\title{
High-grade meningiomas: biology and implications
}

\author{
Wenya Linda Bi, MD, PhD, ${ }^{1}$ Vikram C. Prabhu, MD, ${ }^{2}$ and lan F. Dunn, $\mathrm{MD}^{1}$ \\ ${ }^{1}$ Center for Skull Base and Pituitary Surgery, Department of Neurosurgery, Brigham and Women's Hospital, Harvard Medical \\ School, Boston, Massachusetts; and 'Departments of Neurological Surgery and Radiation Oncology, Loyola University Medical
} Center, Chicago, Illinois

\begin{abstract}
The epochal developments in the treatment of meningioma-microsurgery, skull base techniques, and radiation therapy-will be appended to include the rational application of targeted and immune therapeutics, previously ill-fitting concepts for a tumor that has traditionally been a regarded as a surgical disease. The genomic and immunological architecture of these tumors continues to be defined in ever-greater detail. Grade I meningiomas are driven by NF2 alterations or mutations in AKT1, SMO, TRAF7, PIK3CA, KLF4, POLR2A, SUFU, and SMARCB1. Higher-grade tumors, however, are driven nearly exclusively by NF2/chr22 loss and are marked by infrequent targetable mutations, although they may harbor a greater mutation burden overall. TERT mutations may be more common in tumors that progress in histological grade; SMARCE1 alteration has become a signature of the clear cell subtype; and BAP1 in rhabdoid variants may confer sensitivity to pharmacological inhibition. Compared with grade I meningiomas, the most prominent alteration in grade II and III meningiomas is a significant increase in chromosomal gains and losses, or copy number alterations, which may have behavioral implications. Furthermore, integrated genomic analyses suggest phenotypic subgrouping by methylation profile and a specific role for PRC2 complex activation. Lastly, there exists a complex phylogenetic relationship among recurrent high-grade tumors, which continues to underscore a role for the most traditional therapy in our arsenal: surgery.
\end{abstract}

https://thejns.org/doi/abs/10.3171/2017.12.FOCUS17756

KEY WORDS high-grade meningioma; atypical meningioma; anaplastic meningioma; rhabdoid; genomics; methylation; molecular classification

$\mathrm{M}$ ENINGIOMAS, the most common primary tumors of the central nervous system in adults, have inspired significant advances in the evolution of neurosurgery (the story of meningioma is the story of microneurosurgery) and, more recently, in the application of lessons learned from biology to the management of skull base pathologies. Furthermore, the lessons learned from current study of human tumors-i.e., the elucidation of biology, the role of molecular criteria for diagnosis and prognostication, and the application of precision medicine treatment strategies ${ }^{7}$-are being applied in earnest to meningiomas. Despite such advances, subsets of meningiomas remain refractory to multimodality therapies, including aggressive surgery, radiation therapy, and pharmacological trials.

Meningiomas are classified into 3 grades by the WHO; grade II and III meningiomas are associated with high rates of recurrence and premature mortality. ${ }^{23}$ Grade I meningiomas possess fewer than 4 mitoses per 10 microscopic hpf and encompass 9 histological subtypes (meningothelial, fibrous or fibroblastic, transitional, psammoma- tous, angiomatous, microcystic, secretory, lymphoplasmacyte-rich, and metaplastic). Grade II meningiomas include atypical, clear cell, and chordoid morphological variants and are defined by 4-19 mitoses per $10 \mathrm{hpf}$, brain invasion, or the presence of 3 of 5 histopathological features associated with atypia. ${ }^{23}$ These atypical features include sheet-like growth, spontaneous necrosis, high nuclear-tocytoplasmic ratio, prominent nucleoli, and increased cellularity. The presence of 1 or 2 atypical features in a grade I meningioma, even if these features are not able to promote the tumor to a grade II tumor formally, is associated with an increased risk of progression and recurrence. ${ }^{24}$ Grade III meningiomas have a mitotic index of 20 or greater per $10 \mathrm{hpf}$ and incorporate anaplastic, papillary, and rhabdoid subtypes. Grade III meningiomas inevitably recur within 10 years (usually less) and, as such, are considered malignant..$^{17,25}$

Efforts to mitigate the recurrence of high-grade meningioma have been blunted by a dearth of treatment options and biological targets. Since the initial discovery of neurofibromin (NF2), the causative gene for neurofibromato- 
sis type 2 at $22 \mathrm{q} 12.2$, numerous mutations, deletions, and rearrangements resulting in inactivation of $N F 2$ have been described in meningioma as an oncogenic driver. ${ }^{3,10,13,18,27}$ Additional recurrent somatic mutations in the ubiquitin ligase tumor necrosis factor receptor-associated factor 7 (TRAF7), the pluripotency transcription factor Kruppellike factor 4 (KLF4), the proto-oncogene v-Akt murine thymoma viral oncogene homolog 1 ( $A K T 1)$, the Hedgehog pathway signaling member smoothened (SMO), the oncogene phosphatidylinositol-4,5-bisphosphate 3-kinase catalytic subunit alpha (PIK3CA), the gene for the catalytic subunit of RNA polymerase II (POLR2A), the Switch/Sucrose nonfermentable (SWI-SNF) chromatin-remodeling complex gene $S M A R C B 1$, and others of lower incidence have been identified using next-generation sequencing approaches in sporadic meningiomas, usually in the absence of concurrent NF2 or chromosome 22 alterations (Table 1). ${ }^{1,8,10,13,14,21}$ Furthermore, these recurrent mutations, outside of $N F 2$, are largely observed in grade I meningiomas but rarely in grade II or III meningiomas. ${ }^{8}$

We aim to review the distinct genomic alterations observed in grade II and III meningiomas, discuss their diagnostic and therapeutic implications, and hypothesize on the implications of such molecular alterations for the ontogeny of high-grade meningiomas.

\section{Emerging Themes From Genomic Analyses Higher Rates of Clinically Relevant Genomic Disruption Are Present in Grade II and III Meningiomas}

The most prominent alteration in grade II and III meningiomas, compared with grade I meningiomas, is a significant increase in chromosomal gains and losses, or copy number alterations. Aside from the angiomatous subtype of grade I meningiomas, which harbor multiple polysomies across the genome, grade I sporadic meningiomas, or those not associated with an inherited familial syndrome, typically possess no chromosome alterations outside focal or broad loss on chromosome 22 in $40 \%-70 \%$ of cases. In comparison, high-grade meningiomas are associated with both a significantly higher incidence of chromosome 22 and/or NF2 loss and express incremental loss of chromosomes $1 \mathrm{p}, 6 \mathrm{q}, 10,14 \mathrm{q}$, and 18q with increasing grade (Fig. 1). ${ }^{8,11,23}$

Loss of $1 \mathrm{p}$ is the most frequent copy number alteration, following chromosome 22 loss. Its presence in the chromosomal profile of a meningioma portends more aggressive biological behavior and may call for closer surveillance. Interestingly, chromosome 1 loss is observed significantly less frequently among grade III rhabdoid meningiomas, a particularly aggressive subtype, compared with other high-grade subtypes. ${ }^{8}$ Amplification of chromosomes 1q, $5,7,12,15 \mathrm{q}, 17 \mathrm{q}, 20$, and $21 \mathrm{q}$ is also observed in highgrade meningiomas with variable incidence but occur less frequently overall than deletion events (Fig. 1). ${ }^{8}$ On a focal level, loss of 9p21, harboring the cyclin-dependent inhibitors $C D K N 2 A$ and $C D K N 2 B$, is suggestive of increased aggressive behavior among meningiomas and correlates with decreased survival in anaplastic meningiomas. ${ }^{26}$

Genome disruption, as the aggregate sum of copy number losses and gains, is of clinical interest as it might, in
TABLE 1. Recurrent mutations observed in meningiomas

\begin{tabular}{|c|c|}
\hline $\begin{array}{l}\text { WHO Grade \& } \\
\text { Mutant Gene }\end{array}$ & Comment \\
\hline \multicolumn{2}{|l|}{ I } \\
\hline NF2 & Associated w/ chromosome 22 loss \\
\hline \multicolumn{2}{|l|}{ TRAF7 } \\
\hline \multicolumn{2}{|l|}{ AKT1 } \\
\hline \multicolumn{2}{|l|}{ SMO } \\
\hline \multicolumn{2}{|l|}{ PIK3CA } \\
\hline \multicolumn{2}{|l|}{ KLF4 } \\
\hline \multicolumn{2}{|l|}{ POLR2A } \\
\hline \multicolumn{2}{|l|}{ SMARCB1 } \\
\hline \multicolumn{2}{|l|}{ II } \\
\hline \multicolumn{2}{|l|}{ NF2 } \\
\hline SMARCB1 & Germline \& somatic \\
\hline SMARCE1 & Germline \& somatic; associated w/ clear cell subtype \\
\hline TERT & Associated w/ histological progression in grade \\
\hline \multicolumn{2}{|l|}{ III } \\
\hline \multicolumn{2}{|l|}{ NF2 } \\
\hline BAP1 & Associated w/ rhabdoid subtype \\
\hline TERT & Associated w/ histological progression in grade \\
\hline
\end{tabular}

part, predict the risk of tumor recurrence, independent of meningioma grade and treatment status. ${ }^{4}$ Grade I meningiomas that subsequently recur exhibit a higher burden of genomic disruption than those without subsequent recurrence. ${ }^{8}$ This observation suggests a role for integrating molecular features into the pathologic diagnosis for meningioma to further refine its prognostic value.

Genome disruption also exhibits a strong association with the location of meningiomas, independent of tumor grade (W.L. Bi et al., unpublished data). Midline skull base meningiomas harbor zero or rare copy number alterations, while midline convexity (falcine, parasagittal) meningiomas possess abundant chromosomal alterations, with lateral skull base and lateral convexity meningiomas spanning an intermediate range between these 2 extremes. These patterns echo previous observations that distinct putative driver mutations in meningioma cluster in different anatomical locations, and suggest divergent ontogenies for meningioma.

The mechanism for genome disruption remains to be elucidated, although several observations offer speculation on a distinct role for the widespread recurrent chromosomal alterations observed in high-grade meningioma. First, copy number alterations may precede the acquisition of most mutations, outside of $N F 2$, in high-grade meningiomas. Analysis of serial resections of human meningioma from the same patient reveals that copy number alterations are largely shared across recurrences, whereas mutations are generally unique to a single tumor among multiple recurrences. ${ }^{8}$ Second, meningiomas harbor a high incidence of complex rearrangements that link together geographically distinct regions of the genome. ${ }^{8}$ Since structural alterations result in copy number variation, such complex rearrangements might contribute to a proportion of 

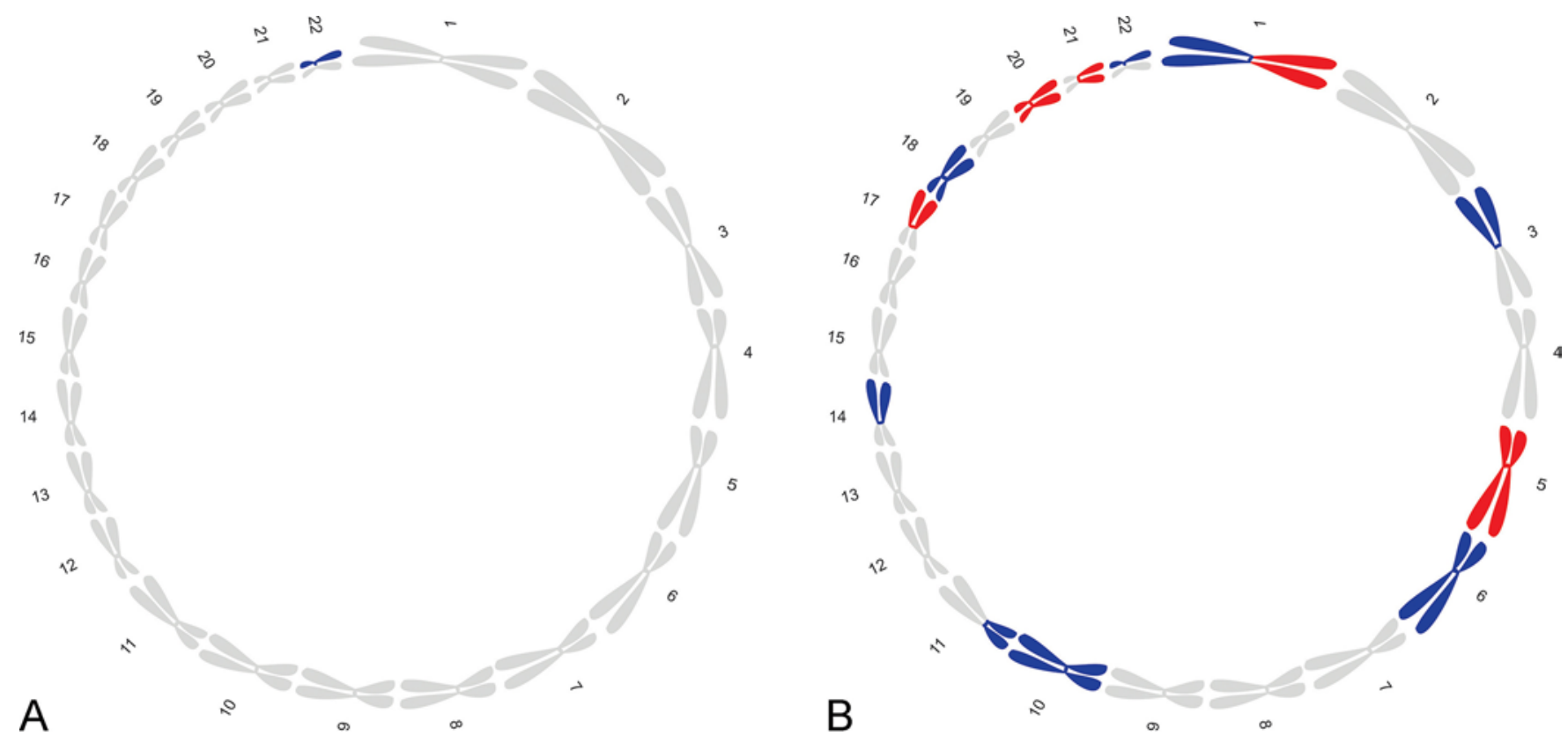

FIG. 1. Chromosomal alterations frequently observed in meningioma. A: Monosomy 22 is the most common copy number alteration across all meningiomas and frequently the only event in grade I meningiomas. B: Recurrent copy number alterations in the colored chromosomes are a hallmark of high-grade meningiomas. Blue denotes chromosomal loss; red, chromosomal gain.

copy number events in select high-grade tumors. Third, distinct pathways may lead to the generation of an atypical meningioma, one involving genome disruption on the background of NF2 loss and another implicating concurrent SMARCB1 mutation with NF2 alteration but without genome disruption. ${ }^{21}$ Meningiomas with concurrent NF2 and SMARCBI alterations demonstrate a predisposition for the falx in some reports, ${ }^{14,32}$ which is also the location associated with the highest burden of genomic disruption across all anatomical regions giving rise to meningioma (W.L. Bi et al., unpublished data). Taken together, the relationship between genomic signature and meningioma origin merits deeper interrogation in future studies.

\section{High-Grade Meningiomas Differ From Grade I Meningiomas in Mutation Profile}

As with genome disruption, high-grade meningiomas are characterized by mutational profiles that differ from those of grade I tumors. High-grade meningiomas express more mutations than grade I meningiomas, on average, ${ }^{8}$ although the number of somatic protein-altering mutations may not significantly differ between grade I and grade II atypical meningiomas. ${ }^{21}$ Increased somatic mutation burden, along with methylation signatures, was associated with decreased overall survival in an integrated molecular analysis of grade I-III meningiomas. ${ }^{33}$

Notably, half of all meningioma mutations are predicted to be neoantigens. High-grade meningiomas, concordantly, have a higher quantity of predicted neoantigens than grade I tumors. Meningiomas offer an advantage, as they are situated outside of the blood-brain barrier and have been demonstrated to attract a robust immune infiltrate and express prominent levels of immune checkpoint markers, especially among high-grade tumors. ${ }^{916,20}$ These qualities suggest a potential role for immunotherapy strategies for select meningioma patients, especially those with high-grade meningiomas with robust mutation burden, which is under clinical trial investigation.

High-grade meningiomas are associated with few specific recurrent somatic mutations outside of $N F 2$, which is in stark contrast to grade I meningioma. ${ }^{8}$ SMARCE1 mutations in clear cell meningioma and $B A P I$ mutation in a subset of rhabdoid meningiomas have been reported, but the majority of high-grade meningiomas have yet to be associated with other putative driver mutations..$^{29-31}$ SMARCBI and other low-incidence mutations are observed in rare grade I and grade II meningiomas, with converging lines of evidence suggestive of a collective role for chromatin regulators in meningioma. ${ }^{10,14,21}$

In meningiomas with demonstrated progression from lower grade to higher grade, mutations of the TERT promoter are observed in one-quarter of cases. ${ }^{19,22}$ In contrast, TERT mutations are not regularly observed among de novo atypical and anaplastic meningiomas or radiation-induced meningiomas. ${ }^{3,8,15,21}$ However, emergence of TERT promoter mutations was detected in regions of a grade III meningioma that were more histologically aggressive than histologically benign areas, a finding that supports differential TERT expression across geographic regions of high-grade tumors, which is a potential confounding influence in the assessment of isolated cores from meningiomas for genomic analysis. ${ }^{2,22}$

\section{Methylation Signatures and Transcriptional Networks Define Subgroups of Meningioma With Prognostic Significance}

Integrated molecular analysis from DNA to RNA to expression and epigenetic markers has revealed additional 
prognostic markers among high-grade meningiomas. Meningiomas of all grades are stratified into 3 major classes based on their methylation profile, with corresponding benign, intermediate, and malignant behavior, with 6 total subclasses. ${ }^{28}$ These methylation divisions associate with specific mutation, cytogenetic, and expression signatures. More importantly, the methylation profile of meningiomas strongly correlates with patient survival, surpassing even the predictive value provided by the current WHO histopathology grade. ${ }^{28}$

In particular, high expression of the transcription factor Forkhead box protein M1 mRNA and protein (encoded by Forkhead box M1 [FOXMI]) was significantly associated with decreased recurrence-free survival across grade I-III meningiomas. ${ }^{33}$ Of note, differential expression of these pathways appears to be independent of copy number variation. Another independent analysis of atypical meningiomas also demonstrated upregulation of the FOXM1 transcriptional network, along with EZH2, the catalytic domain of polycomb repressive complex 2 (PRC2), and the E2F2 transcriptional network. ${ }^{21}$ PRC2 catalyzes transcriptional silencing through methylation and is thought to function in balance with the SWI/SNF complex, which is frequently mutated in anaplastic meningiomas associated with poor prognosis compared with those with more favorable patient survival. ${ }^{15}$ Members of the SWI/SNF complex, including SMARCBI and SMARCE1, are associated with familial syndromes with multiple meningiomas. Specifically, germline mutations in SMARCBI, located at 22q11, have been identified in several families with multiple meningiomas and schwannomas ${ }^{6,12,32}$ as well as in association with grade I and grade II atypical meningiomas. $^{21}$

The enhanced understanding of the epigenome has deepened our understanding of the complexity of highgrade meningioma and may complement genomic approaches in the identification of therapeutic targets.

\section{Meningiomas Exhibit Spatial and Temporal Heterogeneity}

There is an increasing appreciation that genomic profiles may differ within an individual tumor and may change in recurrent specimens; that is, that there is spatial and temporal heterogeneity. Recent data have shown that grade II and III meningiomas are characterized by these features.

Multifocal sampling of regions that appear more histologically benign and areas that possess more histologically aggressive features from a single anaplastic meningioma revealed stepwise acquisition of novel mutations, including ARIDIA (an SWI/SNF complex member) and $T E R T$, where copy number alterations across regions were largely shared. ${ }^{2}$ Such heterogeneity could lead to acquired resistance in patients treated with targeted therapy. Indeed, we have assessed the relationship of different areas of particular tumors and found that only $9 \%$ of mutations were shared between any 2 regions. ${ }^{8}$

Analysis of high-grade meningioma samples reveals that the majority of mutations are not shared across serial recurrences. ${ }^{8}$ This may suggest a proclivity for passenger mutations that are not associated with an oncogenic driver or trigger to arise over time. In comparison, a recurrent high-grade meningioma with multiple chromosomal alter- ations typically possesses a majority of those alterations on first encounter at the primary resection.

One therapeutic implication of intra- and intertumoral heterogeneity and the absence of a dominant clone in recurrent tumors is that aggressive resection will continue to be a critical weapon in our arsenal of treatment options.

\section{Radiation Exerts Unique Influences on the Genomic Profile of Meningioma}

Meningiomas that arise due to distant radiation exposure, or radiation-induced meningiomas, have similar or higher copy number alterations compared with those with no prior radiation exposure..$^{3,8}$ Loss of $1 p$ resulting from deletion or rearrangement, in particular, has been reported at a high incidence among radiation-induced meningiomas, in addition to loss of 22q, even among histologically grade I tumors. ${ }^{3,5,34}$ Radiation-induced meningiomas also possess NF2 fusion events at a striking frequency that is not observed in sporadic meningiomas. ${ }^{3}$

Interestingly, meningiomas exposed to recent adjuvant radiation therapy contained significantly more chromosomal alterations than either radiation-induced meningiomas or radiation-naive meningiomas. ${ }^{8}$ Given the association of genome disruption with long-term recurrence risk, this observation frames deeper exploration for the timing of adjuvant radiation therapy in meningioma.

\section{Conclusions}

The biology of high-grade meningiomas frequently triumphs over existing medical strategies in evading cure. Recurrent mutations observed in grade I meningiomas are largely absent from grade II-III tumors, aside from $N F 2$. In comparison, distinct methylation, gene expression, and copy number profiles mark high-grade meningiomas, affording phenotypic subgroups with prognostic associations and novel therapeutic opportunities. Evolving decryption of the genomic and immunological landscape of these tumors, across space and time, will continue to widen our entourage of pharmacological options in complement to the traditional arsenal of surgery.

\section{References}

1. Abedalthagafi M, Bi WL, Aizer AA, Merrill PH, Brewster R, Agarwalla PK, et al: Oncogenic PI3K mutations are as common as AKT1 and SMO mutations in meningioma. Neuro Oncol 18:649-655, 2016

2. Abedalthagafi MS, Bi WL, Merrill PH, Gibson WJ, Rose MF, Du Z, et al: ARID1A and TERT promoter mutations in dedifferentiated meningioma. Cancer Genet 208:345-350, 2015

3. Agnihotri S, Suppiah S, Tonge PD, Jalali S, Danesh A, Bruce JP, et al: Therapeutic radiation for childhood cancer drives structural aberrations of NF2 in meningiomas. Nat Commun 8:186, 2017

4. Aizer AA, Abedalthagafi M, Bi WL, Horvath MC, Arvold ND, Al-Mefty O, et al: A prognostic cytogenetic scoring system to guide the adjuvant management of patients with atypical meningioma. Neuro Oncol 18:269-274, 2016

5. Al-Mefty O, Topsakal C, Pravdenkova S, Sawyer JR, Harrison MJ: Radiation-induced meningiomas: clinical, pathological, cytokinetic, and cytogenetic characteristics. J Neurosurg 100:1002-1013, 2004 
6. Bacci C, Sestini R, Provenzano A, Paganini I, Mancini I, Porfirio B, et al: Schwannomatosis associated with multiple meningiomas due to a familial SMARCB1 mutation. Neurogenetics 11:73-80, 2010

7. Bi WL, Abedalthagafi M, Horowitz P, Agarwalla PK, Mei Y, Aizer AA, et al: Genomic landscape of intracranial meningiomas. J Neurosurg 125:525-535, 2016

8. Bi WL, Greenwald NF, Abedalthagafi M, Wala J, Gibson WJ, Agarwalla PK, et al: Genomic landscape of high-grade meningiomas. NPJ Genom Med 2:15, 2017

9. Bi WL, Wu WW, Santagata S, Reardon DA, Dunn IF: Checkpoint inhibition in meningiomas. Immunotherapy 8:721-731, 2016

10. Brastianos PK, Horowitz PM, Santagata S, Jones RT, McKenna A, Getz G, et al: Genomic sequencing of meningiomas identifies oncogenic SMO and AKT1 mutations. Nat Genet 45:285-289, 2013

11. Cai DX, Banerjee R, Scheithauer BW, Lohse CM, Kleinschmidt-Demasters BK, Perry A: Chromosome 1p and 14q FISH analysis in clinicopathologic subsets of meningioma: diagnostic and prognostic implications. J Neuropathol Exp Neurol 60:628-636, 2001

12. Christiaans I, Kenter SB, Brink HC, van Os TA, Baas F, van den Munckhof P, et al: Germline SMARCB1 mutation and somatic NF2 mutations in familial multiple meningiomas. J Med Genet 48:93-97, 2011

13. Clark VE, Erson-Omay EZ, Serin A, Yin J, Cotney J, Ozduman K, et al: Genomic analysis of non-NF2 meningiomas reveals mutations in TRAF7, KLF4, AKT1, and SMO. Science 339:1077-1080, 2013

14. Clark VE, Harmancı AS, Bai H, Youngblood MW, Lee TI, Baranoski JF, et al: Recurrent somatic mutations in POLR2A define a distinct subset of meningiomas. Nat Genet 48:12531259,2016

15. Collord G, Tarpey P, Kurbatova N, Martincorena I, Moran S, Castro MR, et al: An integrated genomic analysis of anaplastic meningioma identifies poor prognostic molecular signatures. bioRxiv [epub ahead of print], 2017

16. Du Z, Abedalthagafi M, Aizer AA, McHenry AR, Sun HH, Bray MA, et al: Increased expression of the immune modulatory molecule PD-L1 (CD274) in anaplastic meningioma. Oncotarget 6:4704-4716, 2015

17. Durand A, Labrousse F, Jouvet A, Bauchet L, Kalamaridès M, Menei P, et al: WHO grade II and III meningiomas: a study of prognostic factors. J Neurooncol 95:367-375, 2009

18. Fontaine B, Rouleau GA, Seizinger BR, Menon AG, Jewell AF, Martuza RL, et al: Molecular genetics of neurofibromatosis 2 and related tumors (acoustic neuroma and meningioma). Ann N Y Acad Sci 615:338-343, 1991

19. Goutagny S, Nault JC, Mallet M, Henin D, Rossi JZ, Kalamarides M: High incidence of activating TERT promoter mutations in meningiomas undergoing malignant progression. Brain Pathol 24:184-189, 2014

20. Han SJ, Reis G, Kohanbash G, Shrivastav S, Magill ST, Molinaro AM, et al: Expression and prognostic impact of immune modulatory molecule PD-L1 in meningioma. J Neurooncol 130:543-552, 2016

21. Harmancı AS, Youngblood MW, Clark VE, Coşkun S, Henegariu O, Duran D, et al: Integrated genomic analyses of de novo pathways underlying atypical meningiomas. Nat Commun 8:14433, 2017

22. Juratli TA, Thiede C, Koerner MVA, Tummala SS, Daubner D, Shankar GM, et al: Intratumoral heterogeneity and TERT promoter mutations in progressive/higher-grade meningiomas. Oncotarget 8:109228-109237, 2017

23. Louis D, Ohgaki H, Wiestler OD, Cavenee WK: WHO Classification of Tumours of the Central Nervous System. Lyon: International Agency for Research on Cancer, 2016
24. Marciscano AE, Stemmer-Rachamimov AO, Niemierko A, Larvie M, Curry WT, Barker FG II, et al: Benign meningiomas (WHO Grade I) with atypical histological features: correlation of histopathological features with clinical outcomes. J Neurosurg 124:106-114, 2016

25. Palma L, Celli P, Franco C, Cervoni L, Cantore G: Longterm prognosis for atypical and malignant meningiomas: a study of 71 surgical cases. Neurosurg Focus 2(4):e3, 1997

26. Perry A, Banerjee R, Lohse CM, Kleinschmidt-DeMasters BK, Scheithauer BW: A role for chromosome 9p21 deletions in the malignant progression of meningiomas and the prognosis of anaplastic meningiomas. Brain Pathol 12:183-190, 2002

27. Ruttledge MH, Sarrazin J, Rangaratnam S, Phelan CM, Twist E, Merel P, et al: Evidence for the complete inactivation of the NF2 gene in the majority of sporadic meningiomas. Nat Genet 6:180-184, 1994

28. Sahm F, Schrimpf D, Stichel D, Jones DTW, Hielscher T, Schefzyk S, et al: DNA methylation-based classification and grading system for meningioma: a multicentre, retrospective analysis. Lancet Oncol 18:682-694, 2017

29. Shankar GM, Abedalthagafi M, Vaubel RA, Merrill PH, Nayyar N, Gill CM, et al: Germline and somatic BAP1 mutations in high-grade rhabdoid meningiomas. Neuro Oncol 19:535-545, 2017

30. Smith MJ, O'Sullivan J, Bhaskar SS, Hadfield KD, Poke G, Caird J, et al: Loss-of-function mutations in SMARCE1 cause an inherited disorder of multiple spinal meningiomas. Nat Genet 45:295-298, 2013

31. Tauziede-Espariat A, Parfait B, Besnard A, Lacombe J, Pallud J, Tazi S, et al: Loss of SMARCE1 expression is a specific diagnostic marker of clear cell meningioma: a comprehensive immunophenotypical and molecular analysis. Brain Pathol [epub ahead of print], 2017

32. van den Munckhof P, Christiaans I, Kenter SB, Baas F, Hulsebos TJ: Germline SMARCB1 mutation predisposes to multiple meningiomas and schwannomas with preferential location of cranial meningiomas at the falx cerebri. Neurogenetics 13:1-7, 2012

33. Vasudevan H, Braunstein S, Phillips JJ, Pekmezci M, Wu A, Reis G, et al: Comprehensive genomic characterization of aggressive meningiomas identifies molecular signatures that predict clinical outcomes. Neuro Oncol 19 (Suppl 6):vi92vi93, 2017

34. Zattara-Cannoni H, Roll P, Figarella-Branger D, Lena G, Dufour H, Grisoli F, et al: Cytogenetic study of six cases of radiation-induced meningiomas. Cancer Genet Cytogenet 126:81-84, 2001

\section{Disclosures}

The authors report no conflict of interest concerning the materials or methods used in this study or the findings specified in this paper.

\section{Author Contributions}

Conception and design: Dunn, Bi. Drafting the article: Dunn, Bi. Critically revising the article: all authors. Reviewed submitted version of manuscript: all authors. Approved the final version of the manuscript on behalf of all authors: Dunn.

\section{Correspondence}

Ian F. Dunn: Brigham and Women's Hospital, Boston, MA. idunn@partners.org. 